\title{
Sozialversicherungen in der Schweiz
}

Linus Cavegn, Andy Bühler

Korrespondenz:

FMH Treuhand Services

Geschäftsstelle Basel

Linus Cavegn/Andy Bühler

Hirzbodenweg 103

CH-4020 Basel

Tel. 0613195121

Fax 0613195252

linus.cavegn@fmhtreuhand.ch

\section{Änderungen bei den wichtigsten Eckdaten per 1. Januar 2006}

Im Jahr 2006 wird es bei der Beitragspflicht für die AHV/ALV/IV/EO zu keinen Änderungen kommen. Die aktuellen und historischen Zahlen ersehen Sie in der Tabelle 1.

Neu beitragspflichtig werden Erwerbstätige ab dem Jahrgang 1988.

Die AHV-Renten bleiben gegenüber dem Vorjahr ebenfalls unverändert. Bei den Männern wird der Jahrgang 1941, bei den Frauen der Jahrgang 1942 ordentlich rentenanspruchsberechtigt.

Beim BVG (gesetzliches Minimum) bleiben die gesetzlich vorgeschriebenen Eckwerte unverändert.

Dies gilt ebenfalls für die steuerfreien Grenzbeträge betreffend das Sparen 3a für Arbeitnehmer wie auch für Selbständigerwerbende.

\section{Neue AHV-Nummer}

Die heute gültige 11stellige AHV-Nummer soll durch eine neue 13stellige Nummer ersetzt werden. Es ist vorgesehen, dass die neue Nummer schrittweise ab 1. Juli 2007 angewendet wird. Allen bisher Versicherten wird ebenfalls eine neue 13stellige Nummer zugeteilt. Die alte Num- mer wird im bestehenden AHV-Register mit der neuen verbunden, so dass durch diesen Wechsel keine Daten verlorengehen.

Das aktuelle 11stellige Nummernsystem stösst an seine Grenzen sowohl im Bereich der verwendeten Codierung als auch im Bereich der eindeutigen Zuteilung. Die heutige Nummer enthält verschlüsselte Angaben der Versicherten wie Geburtstag, -monat und -jahr, das Geschlecht und die Anfangsbuchstaben des Geschlechtsnamens sowie ob die Person Schweizer/in oder Ausländer/in ist. Dies ist aus heutiger Sicht heikel in bezug auf die aktuellen Anforderungen des Datenschutzes.

Die neue 13stellige AHV-Nummer ist eine vollständig anonymisierte Nummer, die keinerlei Rückschlüsse auf den Versicherten mehr zulässt. Sie ist eindeutig einer Person zugeordnet und ändert, im Gegensatz zu der bisherigen Nummer, auch nicht bei einem Namenswechsel aufgrund einer Heirat. Sie begleitet den Versicherten somit ein Leben lang.

Die heutige graue AHV-Karte wird durch einen vereinfachten Ausweis ersetzt. Dieser enthält die AHV-Nummer, den Namen, den Vornamen und das Geburtsdatum des Versicherten. 
Tabelle 1

Wichtige Eckdaten (alle Beträge sind in Fr. und verstehen sich als jährliche Ansätze).

\begin{tabular}{|c|c|c|c|c|c|c|c|c|}
\hline & & 2000 & 2001 & 2002 & 2003 & 2004 & 2005 & 2006 \\
\hline \multicolumn{9}{|c|}{$A H V / A L V / I V / E O$} \\
\hline \multicolumn{9}{|c|}{ Beiträge (Arbeitgeber und Arbeitnehmer je 50\%) } \\
\hline \multicolumn{2}{|c|}{$\mathrm{AHV} / \mathrm{IV} / \mathrm{EO}$} & $10,10 \%$ & $10,10 \%$ & $10,10 \%$ & $10,10 \%$ & $10,10 \%$ & $10,10 \%$ & $10,10 \%$ \\
\hline & bis 106800 & $3,00 \%$ & $3,00 \%$ & $3,00 \%$ & $2,50 \%$ & $2,00 \%$ & $2,00 \%$ & $2,00 \%$ \\
\hline & $106801-267000$ & $2,00 \%$ & $2,00 \%$ & $2,00 \%$ & $1,00 \%$ & $0,00 \%$ & $0,00 \%$ & $0,00 \%$ \\
\hline \multirow{2}{*}{\multicolumn{2}{|c|}{$\begin{array}{l}\text { Minimalbeitrag an die AHV für Nichterwerbstätige } \\
\text { Beginn der Beitragspflicht für Erwerbstätige ab Jahrgang (ab 1. Januar) }\end{array}$}} & 390 & 390 & 390 & 425 & 425 & 425 & 425 \\
\hline & & 1982 & 1983 & 1984 & 1985 & 1986 & 1987 & 1988 \\
\hline
\end{tabular}

\section{Grenz- und Freibeträge}

Freibetrag für Personen im Rentenalter (darüber nur AHV-Pflicht, aber keine ALV-Pflicht)

Freibetrag aus Nebenerwerb (mit Verzichtserklärung)*

$\begin{array}{lrrrrrr}16800 & 16800 & 16800 & 16800 & 16800 & 16800 & 16800 \\ 2000 & 2000 & 2000 & 2000 & 2000 & 2000 & 2000\end{array}$

(nur möglich, wenn eine Haupterwerbstätigkeit ausgeübt wird)

* Dabei beurteilt die Ausgleichskasse, was als Nebenerwerb gilt.

Renten

einfache AHV-, IV- und Ehepaarrente (Vollrente gemäss Skala 44)

\begin{tabular}{|c|c|c|c|c|c|c|c|}
\hline Maximum & 24120 & 24720 & 24720 & 25320 & 25320 & 25800 & 25800 \\
\hline Minimum & 12060 & 12360 & 12360 & 12660 & 12660 & 12900 & 12900 \\
\hline Ehepaarrente & 36180 & 37080 & 37080 & 37980 & 37980 & 38700 & 38700 \\
\hline
\end{tabular}

Beginn Rentenanspruchsberechtigung für Jahrgang (jeweils ab 1. des auf den Geburtstag folgenden Monats)

$\begin{array}{lrrrrrrr}\text { Frauen } & 1938 & * & 1939 & 1940 & 1941 & * * & 1942 \\ \text { Männer } & 1935 & 1936 & 1937 & 1938 & 1939 & 1940 & 1941\end{array}$

* Auf den 1. Januar 2001 wurde bei der AHV das Rücktrittsalter für Frauen auf 63 erhöht. Frauen des Jahrgangs 1939 waren als erste von der Erhöhung betroffen. Ihr ordentlicher Rentenanspruch entstand im Jahr 2002.

** Auf den 1. Januar 2005 wurde bei der AHV das Rücktrittsalter für Frauen auf 64 erhöht.

BVG (gesetzliches Minimum)

\begin{tabular}{|c|c|c|c|c|c|c|c|}
\hline Anrechenbares Jahreseinkommen (Maximum) & 72360 & 74160 & 74160 & 75960 & 75960 & 77400 & 77400 \\
\hline Koordinationsabzug & 24120 & 24720 & 24720 & 25320 & 25320 & $22575^{*}$ & $22575^{*}$ \\
\hline Maximaler versicherter Jahreslohn & 48240 & 49440 & 49440 & 50640 & 50640 & 54825 & 54825 \\
\hline Minimaler versicherter Jahreslohn & 3015 & 3090 & 3090 & 3165 & 3165 & 3225 & 3225 \\
\hline Untere Einkommensgrenze & 24120 & 24720 & 24720 & 25320 & 25320 & 19350 & 19350 \\
\hline
\end{tabular}

* $\quad$ 7/8 der maximalen einfachen AHV-Rente.

Sparen $3 a$

steuerfreier Grenzbetrag für Arbeitnehmer oder Selbständigerwerbende mit 2. Säule (BVG): maximal (= $8 \%$ des maximal versicherten BVG-Lohnes)

$\begin{array}{rrrrrrr}5789 & 5933 & 5933 & 6077 & 6077 & 6192 & 6192 \\ 28944 & 29664 & 29664 & 30384 & 30384 & 30960 & 30960\end{array}$

$20 \%$ des Einkommens, aber maximal (= $40 \%$ des maximal

versicherten BVG-Lohnes) 